\title{
Nearly Singular Integrals in 3D Stokes Flow
}

\author{
Svetlana Tlupova ${ }^{1, *}$, J. Thomas Beale ${ }^{2}$ \\ ${ }^{1}$ Department of Mathematics, University of Michigan, Ann Arbor, MI 48109-1043, \\ USA \\ 2 Department of Mathematics, Duke University, Durham, NC 27708-0320, USA
}

\begin{abstract}
A straightforward method is presented for computing three-dimensional Stokes flow, due to forces on a surface, with high accuracy at points near the surface. The flow quantities are written as boundary integrals using the free-space Green's function. To evaluate the integrals near the boundary, the singular kernels are regularized and a simple quadrature is applied in coordinate charts. High order accuracy is obtained by adding special corrections for the regularization and discretization errors, derived here using local asymptotic analysis. Numerical tests demonstrate the uniform convergence rates of the method.
\end{abstract}

AMS subject classifications: 35J47, 65N80, 76D07

Key words: Stokes equations, boundary integral equation method, nearly singular integrals

\section{Introduction}

Low Reynolds number flows are fundamental in a large class of problems, for example, particle and drop motion, the swimming of microorganisms, vesicle flows $[8,14,19,21]$. These phenomena are modeled by the Stokes equations, and a wide variety of numerical techniques have been employed to find solutions, among which boundary integral equation and singularity based methods are most popular. Boundary integral equation methods have several well-known advantages, such as reduction in the dimensionality of the problem and high achievable accuracy of the solution. They have been used effectively to simulate the behavior of drops or vesicles in Stokes flow; comprehensive work includes $[18,21,24]$.

The numerical treatment of integrals similar to the Stokes problem is studied extensively in many works. The discretization of integral equations in three dimensions is usually based on Galerkin or collocation methods, where basis functions are defined and an integration method is used to construct the matrix coefficients. This often involves

*Corresponding author. Email addresses: stlupova@umich.edu (S. Tlupova), beale@math.duke.edu (J. T. Beale) 
a product integration rule or a change of variables. High accuracy solutions can be obtained at points on the boundary and far away from it. When the solution is evaluated on dense grids, the integrals become nearly singular if the evaluation point is close to the surface. This issue is often overlooked; the few works that address it include [2,3,10,12,22]. In [4], partitions of unity were used along with an analytical resolution of the singularity by a change to polar coordinates, in the context of surface scattering problems. These local quadrature methods were extended in [22] to various elliptic problems; for points close to the boundary, interpolation from the solution at far away points was used to achieve higher accuracy.

In Stokes flow simulations, the boundaries, e.g. two drops, often get close to each other, and computing values at nearby points accurately becomes a non-trivial problem. The method of regularization and correction of $[2,3]$ is well suited to handle this difficulty, since it is simple to implement and the work needed does not increase with proximity to the surface. In [2], single and double layer potentials on curves were computed with uniformly high accuracy with respect to the evaluation point. The free-space Green's function is regularized using a small parameter, and the integrals are discretized by a simple quadrature rule. Asymptotic analysis for the integral near the singularity leads to closed form expressions for the leading terms in error due to regularization, and also due to the discretization quadrature. These expressions are then added as corrections to yield higher accuracy of the numerical solution. This work was extended to treatment of double layer potentials on surfaces in 3D in [3]. Two-dimensional boundary integral calculations for a scalar problem from electromagnetics with several boundaries close to each other were done in [23]. In [15], 3D doubly periodic electromagnetic scattering was computed using regularization and corrections for points on the surface.

In this paper, we overcome the issue of near singularity in Stokes flow by extending the method of [3] to evaluate the integrals for velocity and pressure due to forces on closed surfaces in three dimensions. The surface is represented by several overlapping patches, each parametrized in a rectangular system. The method is based on a direct, or Nyström, discretization and partitions of unity, where the Green's function is regularized. To evaluate the integrals near the surface, correction terms for the error are added to achieve high accuracy; these corrections are derived here and in [3] using local asymptotic analysis. The corrections are local and therefore the high order convergence is achieved without increasing the overall computational complexity. Another important aspect of this method is that the integrals are computed at regularly spaced points, without special gridding or cut-off near the singularity as in $[4,22]$, and the spacing does not change when the evaluation point is close to the boundary or on it. This offers a great advantage when solving time-dependent problems, where the integral equations have to be solved at each time step. The specific results are summarized in Theorem 3.1. Numerical examples in Section 6 exhibit third order accuracy, uniformly for points near the surface. 


\section{Mathematical formulation}

In dimensionless form, the incompressible Stokes equations are

$$
-\nabla p+\Delta \mathbf{u}=0, \quad \nabla \cdot \mathbf{u}=0,
$$

where $p$ is the pressure and $\mathbf{u}$ is the flow velocity. One fundamental solution in three dimensions is the Stokeslet and is given by

$$
\mathbf{u}(\mathbf{x})=\frac{\mathbf{f}}{8 \pi|\mathbf{x}|}+\frac{(\mathbf{f} \cdot \mathbf{x}) \mathbf{x}}{8 \pi|\mathbf{x}|^{3}}, \quad p(\mathbf{x})=\frac{\mathbf{f} \cdot \mathbf{x}}{4 \pi|\mathbf{x}|^{3}}
$$

where $\mathbf{x}=(x, y, z)$ and $\mathbf{f}$ is a constant vector. Other fundamental solutions can be derived by differentiating the Stokeslet; see [17] for a comprehensive review. For a volume of fluid bounded by the surface $\partial \Omega$, the velocity $\mathbf{u}(\mathbf{y})$ due to a distribution of forces $\mathbf{f}(\mathbf{x})$ on the surface is the single layer potential obtained by integrating the Stokeslet,

$$
\mathbf{u}(\mathbf{y})=\int_{\partial \Omega} \frac{\mathbf{f}(\mathbf{x})}{8 \pi|\mathbf{y}-\mathbf{x}|}+\frac{(\mathbf{f}(\mathbf{x}) \cdot(\mathbf{y}-\mathbf{x}))(\mathbf{y}-\mathbf{x})}{8 \pi|\mathbf{y}-\mathbf{x}|^{3}} d S(\mathbf{x}), \quad p(\mathbf{y})=\int_{\partial \Omega} \frac{\mathbf{f}(\mathbf{x}) \cdot(\mathbf{y}-\mathbf{x})}{4 \pi|\mathbf{y}-\mathbf{x}|^{3}} d S(\mathbf{x}) .
$$

Boundary integral equation methods are extremely powerful in solving many differential equations, and have been extensively applied to solve Stokes problems $[9,11,16]$. To deal with situations when the velocity is singular (e.g., as an integral over a curve in $\mathbb{R}^{3}$ or points in $\mathbb{R}^{2}$, or over non-smooth surfaces), the method of regularized Stokeslets was introduced in [6]. It is based on smoothing of the forces, and can be used when the force is distributed over a surface as well as over lines and disconnected points in space. The accuracy of the method for surface integrals was analyzed in [7], and demonstrated numerically for a Stokes-Darcy system in 2D in [20]. Evaluating the integrals accurately at points near the boundary remains a challenge, and high resolution or special techniques such as $[2,10]$ have to be used.

In this work, we develop a method for evaluating the Stokes integrals (2.3) in a region in $\mathbb{R}^{3}$ with a smooth boundary. For points near the surface, the integrals become nearly singular, and special care is required to achieve high accuracy. We first rewrite the integrals as follows. Let $G(\mathbf{x})=-1 / 4 \pi|\mathbf{x}|$ be the Green's function of the Laplacian in $\mathbb{R}^{3}$. Noticing that $\nabla G=\mathbf{x} / 4 \pi|\mathbf{x}|^{3}$, and $\mathbf{x} \cdot \nabla G=1 / 4 \pi|\mathbf{x}|$, we write the velocity in (2.2) as

$$
\mathbf{u}(\mathbf{x})=\frac{\mathbf{f}}{2}(\mathbf{x} \cdot \nabla G)+\frac{\mathbf{x}}{2}(\mathbf{f} \cdot \nabla G) .
$$

We use a shorthand notation

$$
\mathbf{u}=\mathbf{F} \cdot \nabla G,
$$

where

$$
\mathbf{F}=\left(\mathbf{F}_{1}, \mathbf{F}_{2}, \mathbf{F}_{3}\right)=\left(f_{1} \mathbf{x}+x_{1} \mathbf{f}, f_{2} \mathbf{x}+x_{2} \mathbf{f}, f_{3} \mathbf{x}+x_{3} \mathbf{f}\right) / 2
$$


Using this, (2.3) becomes

$$
u_{i}(\mathbf{y})=\int_{\partial \Omega} \mathbf{F}_{i}(\mathbf{y}, \mathbf{x}) \cdot \nabla G(\mathbf{y}-\mathbf{x}) d S(\mathbf{x}), \quad p(\mathbf{y})=\int_{\partial \Omega} \mathbf{f}(\mathbf{x}) \cdot \nabla G(\mathbf{y}-\mathbf{x}) d S(\mathbf{x}),
$$

where

$$
\mathbf{F}_{i}(\mathbf{y}, \mathbf{x})=\left(f_{i}(\mathbf{x})(\mathbf{y}-\mathbf{x})+\left(y_{i}-x_{i}\right) \mathbf{f}(\mathbf{x})\right) / 2 .
$$

In the next section, we describe the method of computing the integrals in (2.4) for points y near $\partial \Omega$.

\section{Numerical method}

Consider the integral,

$$
v(\mathbf{y})=\int_{\partial \Omega} \mathbf{f}(\mathbf{x}) \cdot \nabla G(\mathbf{y}-\mathbf{x}) d S(\mathbf{x}),
$$

where $\Omega \subseteq \mathbb{R}^{3}$ is a bounded domain with a smooth boundary $\partial \Omega, G(\mathbf{x})=-1 / 4 \pi|\mathbf{x}|$ is the free-space Green's function for the Laplacian in $\mathbb{R}^{3}$, and $\mathbf{f}$ is a given function on $\partial \Omega$. Integrals of this form are widely used in fluid dynamics, electromagnetics, and other engineering areas. To evaluate (3.1) for $\mathbf{y}$ near the surface, we first split (3.1) into the normal and tangential components. Using the decomposition $\mathbf{f}=(\mathbf{f} \cdot \mathbf{n}) \mathbf{n}-\mathbf{n} \times(\mathbf{n} \times \mathbf{f})$, where $\mathbf{n}$ is the outward unit normal vector, we write (3.1) as

$v(\mathbf{y})=\int_{\partial \Omega}[\mathbf{f}(\mathbf{x}) \cdot \mathbf{n}(\mathbf{x})][\mathbf{n}(\mathbf{x}) \cdot \nabla G(\mathbf{y}-\mathbf{x})] d S(\mathbf{x})+\int_{\partial \Omega}[(\mathbf{f}(\mathbf{x}) \times \mathbf{n}(\mathbf{x}))] \cdot[\nabla G(\mathbf{y}-\mathbf{x}) \times \mathbf{n}(\mathbf{x})] d S(\mathbf{x})$.

We can use identities from the classical potential theory ( [13], [5], Thm 2.1)

$$
\begin{gathered}
\int_{\partial \Omega} \mathbf{n}(\mathbf{x}) \cdot \nabla G(\mathbf{y}-\mathbf{x}) d S(\mathbf{x})=\left\{\begin{aligned}
-1, & \mathbf{y} \in \Omega \\
-1 / 2, & \mathbf{y} \in \partial \Omega \\
0, & \mathbf{y} \in \mathbb{R}^{3} / \bar{\Omega}
\end{aligned}\right. \\
\int_{\partial \Omega} \nabla G(\mathbf{y}-\mathbf{x}) \times \mathbf{n}(\mathbf{x}) d S(\mathbf{x})=0,
\end{gathered}
$$

and rewrite the integrals to reduce the singularity,

$$
v(\mathbf{y})=v^{n}(\mathbf{y})+v^{\tau}(\mathbf{y})
$$

where we defined

$$
\begin{gathered}
v^{n}(\mathbf{y})=\int_{\partial \Omega}\left[\mathbf{f}(\mathbf{x}) \cdot \mathbf{n}(\mathbf{x})-\mathbf{f}\left(\mathbf{x}_{0}\right) \cdot \mathbf{n}\left(\mathbf{x}_{0}\right)\right][\mathbf{n}(\mathbf{x}) \cdot \nabla G(\mathbf{y}-\mathbf{x})] d S(\mathbf{x})-\chi(\mathbf{y}) \mathbf{f}\left(\mathbf{x}_{0}\right) \cdot \mathbf{n}\left(\mathbf{x}_{0}\right), \\
v^{\tau}(\mathbf{y})=\int_{\partial \Omega}\left[\mathbf{f}(\mathbf{x}) \times \mathbf{n}(\mathbf{x})-\mathbf{f}\left(\mathbf{x}_{0}\right) \times \mathbf{n}\left(\mathbf{x}_{0}\right)\right] \cdot[\nabla G(\mathbf{y}-\mathbf{x}) \times \mathbf{n}(\mathbf{x})] d S(\mathbf{x}),
\end{gathered}
$$


with $\chi(\mathbf{x})=1$ for $\mathbf{x} \in \bar{\Omega}$ and 0 otherwise, and $\mathbf{x}_{0}$ is the boundary point closest to $\mathbf{y}$. A numerical method for computing the double layer potential (3.5) with high accuracy in 3D was developed in [3], and it is similar to the method of [2] for evaluating layer potentials near a boundary in 2D. The method consists of replacing the Green's function with a regularized version and discretizing the integrals by a simple quadrature. For points close to the boundary, the integrals are nearly singular, and high accuracy is achieved by adding corrections for the regularization and discretization errors. The Green's function is regularized as

$$
G_{\delta}(\mathbf{x})=G(\mathbf{x}) \operatorname{erf}(|\mathbf{x}| / \delta)=-\operatorname{erf}(|\mathbf{x}| / \delta) / 4 \pi|\mathbf{x}|,
$$

where erf is the error function, so that

$$
\nabla G_{\delta}(\mathbf{y}-\mathbf{x})=\nabla G(\mathbf{y}-\mathbf{x}) \phi(|\mathbf{y}-\mathbf{x}| / \delta),
$$

with a regularization parameter $\delta$ and a shape factor

$$
\phi(r)=\operatorname{erf}(r)-(2 / \sqrt{\pi}) r e^{-r^{2}} .
$$

With $\nabla G$ replaced by $\nabla G_{\delta}$, the integrals are written in a rectangular coordinate system. The surface is parametrized using overlapping coordinate patches $X^{\sigma}: U_{\sigma} \rightarrow \partial \Omega$, where $U_{\sigma}$ is an open subset of $\mathbb{R}^{2}$. We assume that each map $X^{\sigma}: U_{\sigma} \rightarrow \mathbb{R}^{3}$ is smooth and nondegenerate, i.e., $\partial X^{\sigma}(\alpha) / \partial \alpha$ has rank 2 at each point, with $\alpha=\left(\alpha_{1}, \alpha_{2}\right)$. The integrals are then written as sums over the patches using a partition of unity $\left\{\psi^{\sigma}\right\}$,

$$
\int_{\partial \Omega} F(\mathbf{x}) d S(\mathbf{x})=\sum_{\sigma} \int_{U_{\sigma}} F\left(X^{\sigma}(\alpha)\right) \psi\left(X^{\sigma}(\alpha)\right) A^{\sigma}(\alpha) d \alpha,
$$

where $\psi^{\sigma}: \partial \Omega \rightarrow \mathbb{R}$ are smooth functions with compact support in $U_{\sigma}$ that satisfy $\sum_{\sigma} \psi^{\sigma}(\mathbf{x})=$ 1 for each $\mathbf{x} \in \partial \Omega$, and $A^{\sigma}(\alpha) d \alpha$ is the element of surface area in the $\sigma$-th patch. To get the discrete form of the integrals, we choose a grid spacing $h$ on the coordinate patches, so that the $\sigma$-th patch has grid points $\mathbf{x}_{j}^{\sigma}=X^{\sigma}(j h)$ for $j \in \mathbb{Z}^{2}$ with $\mathbf{x}_{j}^{\sigma} \in V_{\sigma}$, where $V_{\sigma}$ is the interior of the support of $\psi^{\sigma}$. We then assume $\mathbf{y}=\mathbf{x}_{0}+b \mathbf{n}_{0}$, for a point $\mathbf{x}_{0}$ on the surface, with some $b$ and $\mathbf{n}_{0}=\mathbf{n}\left(\mathbf{x}_{0}\right)$. The integrals in (3.5) and (3.6) are now approximated by the corresponding sums

$$
\begin{gathered}
S^{n}=\sum_{j, \sigma}\left[\mathbf{f}\left(\mathbf{x}_{j}^{\sigma}\right) \cdot \mathbf{n}\left(\mathbf{x}_{j}^{\sigma}\right)-\mathbf{f}_{0} \cdot \mathbf{n}_{0}\right]\left[\mathbf{n}\left(\mathbf{x}_{j}^{\sigma}\right) \cdot \nabla G_{\delta}\left(\mathbf{y}-\mathbf{x}_{j}^{\sigma}\right)\right] \psi_{j}^{\sigma} A_{j}^{\sigma} h^{2}-\chi(\mathbf{y}) \mathbf{f}_{0} \cdot \mathbf{n}_{0}, \\
S^{\tau}=\sum_{j, \sigma}\left[\mathbf{f}\left(\mathbf{x}_{j}^{\sigma}\right) \times \mathbf{n}\left(\mathbf{x}_{j}^{\sigma}\right)-\mathbf{f}_{0} \times \mathbf{n}_{0}\right] \cdot\left[\nabla G_{\delta}\left(\mathbf{y}-\mathbf{x}_{j}^{\sigma}\right) \times \mathbf{n}\left(\mathbf{x}_{j}^{\sigma}\right)\right] \psi_{j}^{\sigma} A_{j}^{\sigma} h^{2},
\end{gathered}
$$

where $\mathbf{f}_{0}=\mathbf{f}\left(\mathbf{x}_{0}\right), \psi_{j}^{\sigma}=\psi^{\sigma}\left(\mathbf{x}_{j}^{\sigma}\right)$, and $A_{j}^{\sigma} h^{2}=A^{\sigma}(j h) h^{2}$ is the discrete element of surface area in the patch $\sigma$. The error in the sums (3.9) and (3.10) is large for $\mathbf{y}$ near $\partial \Omega$ and has two parts, the regularization error from replacing $\nabla G$ with $\nabla G_{\delta}$, and the discretization error from replacing the integrals with $\nabla G_{\delta}$ by the sums. It was shown in [3] that these 
errors for the double layer sum (3.9) are $O\left(\delta^{2}\right)$ and $O(h)$, respectively, and corrections were derived to increase the accuracy to $O\left(\delta^{3}\right)$ and at least $O\left(h^{2}\right)$, uniform with respect to location; see Theorem 3.1 below. We state the corrections for the double layer sum (3.9) here for completeness and refer the reader to [3] for the derivation. We then give corrections for the sum (3.10), which will be derived in this paper.

The corrections are derived by asymptotic analysis near $\mathbf{x}_{0}$, and therefore involve the surface geometry there. There is at least one $\sigma$ so that $\mathbf{x}_{0}$ is in the $\sigma$ th patch; i.e., $\mathbf{x}_{0}=X^{\sigma}\left(\alpha_{0}\right)$ for some $\alpha_{0} \in U_{\sigma}$. Let $\mathbf{T}_{i}$ be the tangent vector $\left(\partial X^{\sigma} / \partial \alpha_{i}\right)\left(\alpha_{0}\right)$ at $\mathbf{x}_{0}, i=1,2$, and $\mathbf{n}=\mathbf{T}_{1} \times \mathbf{T}_{2} /\left|\mathbf{T}_{1} \times \mathbf{T}_{2}\right|$ be the unit normal. The regularization correction represents the largest error in regularizing the kernel in (3.5), and is given by

$$
\mathcal{N}_{1}=-\delta^{2}\left(\Delta(\mathbf{f} \cdot \mathbf{n})\left(\mathbf{x}_{0}\right)\right)(\lambda / 4)\left(|\lambda| \operatorname{erfc}|\lambda|-e^{-\lambda^{2}} / \sqrt{\pi}\right),
$$

where $\lambda=b / \delta$, erfc is the complementary error function, and the surface Laplacian is defined as

$$
\Delta w=\sum_{i, j=1}^{2} \frac{1}{\sqrt{g}} \frac{\partial}{\partial \alpha_{j}}\left(\sqrt{g} g g^{i j} \frac{\partial\left(w \circ X^{\sigma}\right)}{\partial \alpha_{i}}\right),
$$

where the metric tensor $g_{i j}=\mathbf{T}_{i} \cdot \mathbf{T}_{j}$, its determinant $g=\operatorname{det}\left(g_{i j}\right)$, and the inverse $g^{i j}=\left(g_{i j}\right)^{-1}$ are used. The discretization correction comes from the Poisson summation formula applied to the regularized kernel, and is computed for every patch that $\mathbf{x}_{0}$ belongs to. In the $\sigma$ th patch, $\mathbf{x}_{0}=X^{\sigma}\left(\alpha_{0}\right)$ for some $\alpha_{0}$ depending on $\sigma$. Let $\alpha_{0}=i h+v h$ for some $i \in \mathbb{Z}^{2}$ and $v=\left(v_{1}, v_{2}\right)$, with $0 \leq v_{s} \leq 1, s=1,2$. The correction for $\sigma$ th patch is [3]

$$
\begin{gathered}
\mathcal{N}_{2}^{\sigma}=h \sum_{r=1}^{2} c_{r} \psi^{\sigma}\left(\alpha_{0}\right) \frac{\partial\left((\mathbf{f} \cdot \mathbf{n}) \circ X^{\sigma}\right)}{\partial \alpha_{r}}\left(\alpha_{0}\right), \\
c_{r}=\frac{\rho \lambda}{2} \sum_{s=1}^{2} \sum_{n \in Q} \sin (2 \pi n \cdot v) \frac{g^{r s} n_{s}}{\|n\|} E(\lambda, \pi \rho\|n\|),
\end{gathered}
$$

where $Q=\left\{n=\left(n_{1}, n_{2}\right) \in \mathbb{Z}^{2}: n_{2}>0\right.$ or $\left(n_{2}=0\right.$ and $\left.\left.n_{1}>0\right)\right\}, \rho=\delta / h,\|n\|=\sqrt{g^{i j} n_{i} n_{j}}$, and

$$
E(a, b)=e^{2 a b} \operatorname{erfc}(a+b)+e^{-2 a b} \operatorname{erfc}(-a+b) .
$$

The sum converges very rapidly provided $\rho$ is not small, so that only a few terms are needed. These correction formulas correspond to (1.21)-(1.25) in [3].

In the following sections, we compute correction terms for the tangential sum (3.10) to achieve the same accuracy in the combined integral. The correction for regularization is

$$
\mathcal{T}_{1}=\frac{\delta}{2}(1+H \lambda \delta)(\nabla \cdot \mathbf{f})\left(\mathbf{x}_{0}\right)\left(|\lambda| \operatorname{erfc}|\lambda|-e^{-\lambda^{2}} / \sqrt{\pi}\right) .
$$

Here $H$ is the mean curvature at $\mathbf{x}_{0}, H=\left(\kappa_{1}+\kappa_{2}\right) / 2$, where $\kappa_{1}, \kappa_{2}$ are the principal curvatures. We compute the mean curvature as $2 H=\sum_{i, j=1}^{2} g^{i j} L_{i j}$, where $L_{i j}=\mathbf{x}_{i j} \cdot \mathbf{n}$ is the second 
fundamental form, and $\mathbf{x}_{i j}=\partial^{2} X^{\sigma} / \partial \alpha_{i} \partial \alpha_{j}$ ( [1], Sec. 9.37). Also, we compute the surface divergence as ( [1], (9.41.1))

$$
\nabla \cdot \mathbf{f}=\sum_{i=1}^{2} \frac{1}{\sqrt{g}} \frac{\partial}{\partial \alpha_{i}}\left(\sqrt{g} f^{(i)}\right)
$$

where $f^{(i)}=\left(\mathbf{f} \circ X^{\sigma}\right) \cdot \mathbf{T}_{i}^{*}$, with $\mathbf{T}_{i}^{*}$ being the dual basis to $\mathbf{T}_{i}$. The correction for discretization error is

$$
\mathcal{T}_{2}^{\sigma}=h \sum_{j, r=1}^{2} c_{j, r} \psi^{\sigma}\left(\alpha_{0}\right) F_{r}^{j}\left(\alpha_{0}\right), \quad F_{r}^{j}=\frac{\partial}{\partial \alpha_{r}}\left(f^{(1)} \mathbf{T}_{1}+f^{(2)} \mathbf{T}_{2}\right) \cdot \mathbf{T}_{j}^{*},
$$

with

$$
\begin{aligned}
c_{j, r} & =\frac{1}{2} \sum_{n \in Q} \cos (2 \pi n \cdot v)\left[\frac{\delta_{j r}}{2 \pi\|n\|} E(\lambda, \pi \rho\|n\|)-\frac{n_{j}}{2 \pi\|n\|^{2}}\left(\sum_{s=1}^{2} g^{r s} \frac{n_{s}}{\|n\|}\right) E(\lambda, \pi \rho\|n\|)\right. \\
& \left.+\frac{n_{j}}{\|n\|}\left(\sum_{s=1}^{2} g^{r s} \frac{n_{s}}{\|n\|}\right) \lambda \rho E_{-}(\lambda, \pi \rho\|n\|)-\frac{n_{j}}{\|n\|}\left(\sum_{s=1}^{2} g^{r s} \frac{n_{s}}{\|n\|}\right) \frac{2 \rho}{\sqrt{\pi}} e^{-\lambda^{2}} e^{-\pi^{2}\|n\|^{2} \rho^{2}}\right],
\end{aligned}
$$

where $E$ is defined as in (3.15), and

$$
E_{-}(a, b)=e^{2 a b} \operatorname{erfc}(a+b)-e^{-2 a b} \operatorname{erfc}(-a+b) .
$$

We can rewrite $F_{r}^{j}$ using Christoffel symbols as

$$
F_{r}^{j}=\frac{\partial f^{(j)}}{\partial \alpha_{r}}+f^{(1)} \Gamma_{1 r}^{j}+f^{(2)} \Gamma_{2 r}^{j}
$$

Theorem 3.1. Let $v(\mathbf{y})$ be given by (3.4), (3.5), (3.6), with $\mathbf{f}$ and $\partial \Omega$ both smooth, and let $\tilde{v}(\mathbf{y})$ be an approximation found as

$$
\tilde{v}(\mathbf{y})=S^{n}+S^{\tau}+\mathcal{N}_{1}+\mathcal{T}_{1}+\sum_{\sigma} \mathcal{N}_{2}^{\sigma}+\sum_{\sigma} \mathcal{T}_{2}^{\sigma}
$$

where $S^{n}, S^{\tau}, \mathcal{N}_{1}, \mathcal{T}_{1}, \mathcal{N}_{2}^{\sigma}$, and $\mathcal{T}_{2}^{\sigma}$ are given by (3.9), (3.10), (3.11), (3.16), (3.13), and (3.18), respectively. Then the error has the form $\tilde{v}(\mathbf{y})-v(\mathbf{y})=\epsilon_{1}+\epsilon_{2}$, where the regularization error $\epsilon_{1}$ and the discretization error $\epsilon_{2}$ can be estimated as

$$
\left|\epsilon_{1}\right| \leq C_{1} \delta^{3}, \quad\left|\epsilon_{2}\right| \leq C_{2} h^{2} e^{-c_{0}(\delta / h)^{2}}+C_{3} h^{3},
$$

uniformly for $\mathbf{y}$ near $\partial \Omega$, where $c_{0}$ depends only on the coordinate patches.

These error estimates were shown in [3] for the normal component $v^{n}$, defined in (3.5) (also see [2] for the two-dimensional case). We follow the same approach and prove the theorem for the tangential component $v^{\tau}$ in (3.6). 


\section{Correction for regularization}

In this section we derive the correction to the error in the integral (3.6), due to regularizing the Green's function as in (3.7). The correction includes the $O(\delta)$ and $O\left(\delta^{2}\right)$ terms, and the remainder is $O\left(\delta^{3}\right)$. Since the error is localized, we assume that $\mathbf{f}=0$ outside one coordinate patch, and write the error as an integral in this patch,

$$
\epsilon=\int\left[\mathbf{f}(\alpha) \times \mathbf{n}(\alpha)-\mathbf{f}_{0} \times \mathbf{n}_{0}\right] \cdot\left[\nabla\left(G_{\delta}-G\right)(\mathbf{y}-\mathbf{x}(\alpha)) \times \mathbf{n}(\alpha)\right] d S(\alpha) .
$$

Assume that $\mathbf{x}(0)=\mathbf{0}$ and $\mathbf{y}$ is along the normal line from $\mathbf{x}(0)$, so that $\mathbf{y}=b \mathbf{n}_{0}$ for some $b$, where $\mathbf{n}_{0}$ is the unit normal at $\mathbf{x}(0)$.

Proposition 4.1. Let $\epsilon$ be the regularization error defined in (4.1). Then $\epsilon=-\mathcal{T}_{1}+O\left(\delta^{3}\right)$, as $\delta \rightarrow 0$, with $\mathcal{T}_{1}$ given by (3.16).

Proof. We assume that $\mathbf{f}$ has the following components,

$$
\mathbf{f}(\alpha)=f^{(1)}(\alpha) \mathbf{T}_{1}(\alpha)+f^{(2)}(\alpha) \mathbf{T}_{2}(\alpha)+f^{(n)}(\alpha) \mathbf{n}(\alpha),
$$

where $f^{(n)}=\mathbf{f} \cdot \mathbf{n}$ and $f^{(i)}=\mathbf{f} \cdot \mathbf{T}_{i}^{*}$, with $\mathbf{T}_{i}^{*}$ the dual basis to $\mathbf{T}_{i}$. The normal component $f^{(n)}$ contributes zero in the cross product, and we denote the tangential component by $\mathbf{f}^{T}=f^{(1)} \mathbf{T}_{1}+f^{(2)} \mathbf{T}_{2}$. Since

$$
\frac{1}{r} \frac{\partial}{\partial r}\left(G_{\delta}-G\right)=\frac{1}{4 \pi r^{3}} \phi\left(\frac{r}{\delta}\right)
$$

we can write

$$
\epsilon=\frac{1}{4 \pi} \int\left[\mathbf{f}^{T}(\alpha) \times \mathbf{n}(\alpha)-\mathbf{f}_{0}^{T} \times \mathbf{n}_{0}\right] \cdot\left[\frac{(\mathbf{y}-\mathbf{x}(\alpha)) \times \mathbf{n}(\alpha)}{r^{3}}\right] \phi\left(\frac{r}{\delta}\right) d S(\alpha),
$$

where $r=|\mathbf{y}-\mathbf{x}(\alpha)|$. As in [3], the dominant contribution to $\epsilon$ is found using Taylor expansions at $\alpha=0$. For simplicity, this is done first for a specially chosen coordinate system and then extended to a general system. With tangent vectors to surface $\mathbf{T}_{j}=$ $\partial \mathbf{x} / \partial \alpha_{j}, j=1,2$ at $\alpha=0$, assume the metric tensor $g_{i j}=\mathbf{T}_{i} \cdot \mathbf{T}_{j}$ is the identity, and $\partial g_{i j} / \partial \alpha_{k}=0$ at $\alpha=0, i, j, k=1,2$. Also, rotating if necessary, $\mathbf{T}_{1}, \mathbf{T}_{2}$ have the directions of principal curvature, and $\mathbf{n}_{0}=\mathbf{T}_{1} \times \mathbf{T}_{2}$. Then, using the expansions

$$
\begin{aligned}
& \mathbf{x}(\alpha)=\mathbf{T}_{1} \alpha_{1}+\mathbf{T}_{2} \alpha_{2}+\frac{1}{2} \kappa_{1} \mathbf{n}_{0} \alpha_{1}^{2}+\frac{1}{2} \kappa_{2} \mathbf{n}_{0} \alpha_{2}^{2}+O\left(|\alpha|^{3}\right), \\
& \mathbf{n}(\alpha)=\mathbf{n}_{0}-\kappa_{1} \mathbf{T}_{1} \alpha_{1}-\kappa_{2} \mathbf{T}_{2} \alpha_{2}+O\left(|\alpha|^{2}\right)
\end{aligned}
$$

where $\kappa_{1}, \kappa_{2}$ are the principal curvatures, we get

$$
(\mathbf{y}-\mathbf{x}(\alpha)) \times \mathbf{n}(\alpha)=-\left(1-b \kappa_{2}\right) \alpha_{2} \mathbf{T}_{1}+\left(1-b \kappa_{1}\right) \alpha_{1} \mathbf{T}_{2}-\left(\kappa_{1}-\kappa_{2}\right) \alpha_{1} \alpha_{2} \mathbf{n}_{0}+O\left(|\alpha|^{3}+b^{3}\right) .
$$


Using the Taylor expansion of $\mathbf{f}^{T}$,

$$
\begin{aligned}
\mathbf{f}^{T}(\alpha) \times \mathbf{n}(\alpha)-\mathbf{f}_{0}^{T} \times \mathbf{n}_{0} & =\left[f^{(1)}+f_{i}^{(1)} \alpha_{i}+\frac{1}{2} f_{i j}^{(1)} \alpha_{i} \alpha_{j}+O\left(|\alpha|^{3}\right)\right] \mathbf{T}_{1}(\alpha) \times \mathbf{n}(\alpha) \\
& +\left[f^{(2)}+f_{i}^{(2)} \alpha_{i}+\frac{1}{2} f_{i j}^{(2)} \alpha_{i} \alpha_{j}+O\left(|\alpha|^{3}\right)\right] \mathbf{T}_{2}(\alpha) \times \mathbf{n}(\alpha) \\
& -\left[f^{(1)} \mathbf{T}_{1}+f^{(2)} \mathbf{T}_{2}\right] \times \mathbf{n}_{0} \\
= & -\left[f_{i}^{(1)} \alpha_{i}+\frac{1}{2} f_{i j}^{(1)} \alpha_{i} \alpha_{j}\right] \mathbf{T}_{2}+\left[f_{i}^{(2)} \alpha_{i}+\frac{1}{2} f_{i j}^{(2)} \alpha_{i} \alpha_{j}\right] \mathbf{T}_{1} \\
& -\left[f^{(1)}+f_{i}^{(1)} \alpha_{i}\right] \kappa_{2} \mathbf{n}_{0} \alpha_{2}+\left[f^{(2)}+f_{i}^{(2)} \alpha_{i}\right] \kappa_{1} \mathbf{n}_{0} \alpha_{1}+O\left(|\alpha|^{3}\right),
\end{aligned}
$$

where $f_{i}^{(k)}=\partial f^{(k)} / \partial \alpha_{i}(0)$ and $f_{i j}^{(k)}=\partial^{2} f^{(k)} / \partial \alpha_{i} \partial \alpha_{j}(0)$. Combining (4.3) and (4.4), we get

$$
\begin{aligned}
& {\left[\mathbf{f}^{T}(\alpha) \times \mathbf{n}(\alpha)-\mathbf{f}_{0}^{T} \times \mathbf{n}_{0}\right] \cdot[(\mathbf{y}-\mathbf{x}(\alpha)) \times \mathbf{n}(\alpha)] } \\
= & -\left(1-b \kappa_{1}\right) \alpha_{1}\left[f_{i}^{(1)} \alpha_{i}+\frac{1}{2} f_{i j}^{(1)} \alpha_{i} \alpha_{j}\right]-\left(1-b \kappa_{2}\right) \alpha_{2}\left[f_{i}^{(2)} \alpha_{i}+\frac{1}{2} f_{i j}^{(2)} \alpha_{i} \alpha_{j}\right] \\
& +f^{(1)}\left(\kappa_{1}-\kappa_{2}\right) \kappa_{2} \alpha_{1} \alpha_{2}^{2}-f^{(2)}\left(\kappa_{1}-\kappa_{2}\right) \kappa_{1} \alpha_{1}^{2} \alpha_{2}+O\left(|\alpha|^{4}+b^{4}\right) .
\end{aligned}
$$

We define a new parameter $\xi$ to replace $\alpha$ so that $r^{2}=b^{2}+|\xi|^{2}$ and $\xi_{i} /|\xi|=\alpha_{i} /|\alpha|$. Then

$$
\begin{gathered}
\alpha_{j}=\left(1+\frac{b q}{2}\right) \xi_{j}+O\left(|\xi|^{3}\right)+O\left(b^{3}\right), \\
q=\kappa_{1} \frac{\xi_{1}^{2}}{|\xi|^{2}}+\kappa_{2} \frac{\xi_{2}^{2}}{|\xi|^{2}}
\end{gathered}
$$

near $\alpha=0$ (see [3]). The regularization error is now written as

$$
\epsilon=\frac{1}{4 \pi} \int w(\xi, b) \frac{\phi\left(\sqrt{|\xi|^{2}+b^{2}} / \delta\right)}{\left(|\xi|^{2}+b^{2}\right)^{3 / 2}} d \xi,
$$

where $w(\xi, b)$ includes the nonradial terms,

$$
w(\xi, b)=\left[\mathbf{f}^{T} \times \mathbf{n}-\mathbf{f}_{0}^{T} \times \mathbf{n}_{0}\right] \cdot[(\mathbf{y}-\mathbf{x}) \times \mathbf{n}]\left|\frac{\partial \alpha}{\partial \xi}\right|\left|\mathbf{T}_{1} \times \mathbf{T}_{2}\right| .
$$

Using (4.5), we get in the new parameter

$$
\begin{aligned}
{\left[\mathbf{f}^{T} \times \mathbf{n}-\mathbf{f}_{0}^{T} \times \mathbf{n}_{0}\right] \cdot[(\mathbf{y}-\mathbf{x}) \times \mathbf{n}]=} & -\left(1+b q-b \kappa_{1}\right) f_{i}^{(1)} \xi_{i} \xi_{1}-\frac{1}{2} f_{i j}^{(1)} \xi_{i} \xi_{j} \xi_{1} \\
& -\left(1+b q-b \kappa_{2}\right) f_{i}^{(2)} \xi_{i} \xi_{2}-\frac{1}{2} f_{i j}^{(2)} \xi_{i} \xi_{j} \xi_{2} \\
& +f^{(1)}\left(\kappa_{1}-\kappa_{2}\right) \kappa_{2} \xi_{1} \xi_{2}^{2}-f^{(2)}\left(\kappa_{1}-\kappa_{2}\right) \kappa_{1} \xi_{1}^{2} \xi_{2}+O\left(|\xi|^{4}+b^{4}\right) .
\end{aligned}
$$


Since $q$ depends only on $\xi /|\xi|$, for the Jacobian we have $|\partial \alpha / \partial \xi|=1+b q+O\left(|\xi|^{2}+b^{2}\right)$, and also $\left|\mathbf{T}_{1} \times \mathbf{T}_{2}\right|=1+O\left(|\xi|^{2}\right)$. Therefore,

$$
\begin{aligned}
w(\xi, b)= & -\left(1+2 b q-b \kappa_{1}\right) f_{i}^{(1)} \xi_{i} \xi_{1}-\frac{1}{2} f_{i j}^{(1)} \xi_{i} \xi_{j} \xi_{1} \\
& -\left(1+2 b q-b \kappa_{2}\right) f_{i}^{(2)} \xi_{i} \xi_{2}-\frac{1}{2} f_{i j}^{(2)} \xi_{i} \xi_{j} \xi_{2} \\
& +f^{(1)}\left(\kappa_{1}-\kappa_{2}\right) \kappa_{2} \xi_{1} \xi_{2}^{2}-f^{(2)}\left(\kappa_{1}-\kappa_{2}\right) \kappa_{1} \xi_{1}^{2} \xi_{2}+O\left(|\xi|^{4}+b^{4}\right) .
\end{aligned}
$$

The only terms in $w(\xi, b)$ that make non-zero contribution to the integral have even powers,

$$
w^{e v e n}(\xi, b)=-\left(1+2 b q-b \kappa_{1}\right) f_{1}^{(1)} \xi_{1}^{2}-\left(1+2 b q-b \kappa_{2}\right) f_{2}^{(2)} \xi_{2}^{2}+O\left(|\xi|^{4}+b^{4}\right) .
$$

Substituting (4.7) into (4.6), and making the substitutions $\xi=\delta \zeta, b=\delta \lambda,|\zeta|=\eta, q=$ $\kappa_{1} \cos ^{2} \theta+\kappa_{2} \sin ^{2} \theta$, we get

$$
\begin{aligned}
\epsilon & =-\frac{1}{4 \pi} \int_{0}^{2 \pi} \int_{0}^{\infty}\left[\left(1+2 b \kappa_{1} \cos ^{2} \theta+2 b \kappa_{2} \sin ^{2} \theta-b \kappa_{1}\right) f_{1}^{(1)} \eta^{2} \cos ^{2} \theta\right. \\
& \left.+\left(1+2 b \kappa_{1} \cos ^{2} \theta+2 b \kappa_{2} \sin ^{2} \theta-b \kappa_{2}\right) f_{2}^{(2)} \eta^{2} \sin ^{2} \theta\right] \delta \frac{\phi\left(\sqrt{\eta^{2}+\lambda^{2}}\right)}{\left(\eta^{2}+\lambda^{2}\right)^{3 / 2}} \eta d \eta d \theta+O\left(\delta^{3}\right),
\end{aligned}
$$

which simplifies to

$$
\epsilon=-\frac{\delta}{4}\left(1+\frac{1}{2}\left(\kappa_{1}+\kappa_{2}\right) \lambda \delta\right)\left(f_{1}^{(1)}+f_{2}^{(2)}\right) \int_{0}^{\infty} \eta^{3} \frac{\phi\left(\sqrt{\eta^{2}+\lambda^{2}}\right)}{\left(\eta^{2}+\lambda^{2}\right)^{3 / 2}} d \eta+O\left(\delta^{3}\right) .
$$

The integral in (4.8) was computed in [3], to give

$$
\int_{0}^{\infty} \eta^{3} \frac{\phi\left(\sqrt{\eta^{2}+\lambda^{2}}\right)}{\left(\eta^{2}+\lambda^{2}\right)^{3 / 2}} d \eta=2\left(|\lambda| \operatorname{erfc}|\lambda|-e^{-\lambda^{2}} / \sqrt{\pi}\right)
$$

Finally,

$$
\epsilon=-\frac{\delta}{2}\left(1+\frac{1}{2}\left(\kappa_{1}+\kappa_{2}\right) \lambda \delta\right)\left(f_{1}^{(1)}+f_{2}^{(2)}\right)\left(|\lambda| \operatorname{erfc}|\lambda|-e^{-\lambda^{2}} / \sqrt{\pi}\right)+O\left(\delta^{3}\right) .
$$

This gives the correction expressed in a special coordinate system. It is extended to an arbitrary system using the surface divergence operator given by (3.17). In the special system at $\alpha=0$, it reduces to $f_{1}^{(1)}+f_{2}^{(2)}$. Therefore, $\epsilon=-\mathcal{T}_{1}+O\left(\delta^{3}\right)$, where $\mathcal{T}_{1}$ is given by (3.16).

\section{Correction for quadrature}

For the discretization error in (3.6), start by adding and subtracting $\mathbf{f}^{T}\left(\mathbf{x}_{0}\right) \times \mathbf{n}(\mathbf{x})$,

$$
I=\int_{\partial \Omega}\left[\mathbf{f}^{T}(\mathbf{x}) \times \mathbf{n}(\mathbf{x})-\mathbf{f}^{T}\left(\mathbf{x}_{0}\right) \times \mathbf{n}(\mathbf{x})+\mathbf{f}^{T}\left(\mathbf{x}_{0}\right) \times \mathbf{n}(\mathbf{x})-\mathbf{f}^{T}\left(\mathbf{x}_{0}\right) \times \mathbf{n}\left(\mathbf{x}_{0}\right)\right] \cdot\left[\nabla G_{\delta}(\mathbf{y}-\mathbf{x}) \times \mathbf{n}(\mathbf{x})\right] d S(\mathbf{x}),
$$


which we write as

$$
I=\int_{\partial \Omega}\left[\left(\mathbf{f}^{T}(\alpha)-\mathbf{f}_{0}^{T}\right) \times \mathbf{n}(\alpha)+\mathbf{f}_{0}^{T} \times\left(\mathbf{n}(\alpha)-\mathbf{n}_{0}\right)\right] \cdot\left[\nabla G_{\delta}(\mathbf{y}-\mathbf{x}(\alpha)) \times \mathbf{n}(\alpha)\right] \tau(\alpha) d \alpha,
$$

where $\mathbf{f}^{T}=\sum_{j=1}^{2} f^{(j)} \mathbf{T}_{j}$ is the tangential component of $\mathbf{f}, \tau(\alpha)=\left|\mathbf{T}_{1}(\alpha) \times \mathbf{T}_{2}(\alpha)\right|$, and $\mathbf{n}(\alpha)=$ $\left(\mathbf{T}_{1}(\alpha) \times \mathbf{T}_{2}(\alpha)\right) / \tau(\alpha)$. In discretizing (5.1), the largest contribution to the error comes from the lowest order in $\alpha$ and $\mathbf{y}$ near the singularity, so we first simplify the integrand. We replace $\mathbf{f}^{T}-\mathbf{f}_{0}^{T}=\mathbf{f}^{T}(\alpha)-\mathbf{f}^{T}(0)$ and $\mathbf{n}-\mathbf{n}_{0}=\mathbf{n}(\alpha)-\mathbf{n}(0)$ by their linear approximations,

$$
\begin{gathered}
\mathbf{f}^{T}(\alpha)-\mathbf{f}^{T}(0) \approx \sum_{r=1}^{2} \frac{\partial \mathbf{f}^{T}}{\partial \alpha_{r}} \alpha_{r} \equiv \mathbf{q}_{1}, \\
\mathbf{n}(\alpha)-\mathbf{n}(0) \approx \sum_{r=1}^{2} \frac{\partial \mathbf{n}}{\partial \alpha_{r}} \alpha_{r} \equiv \mathbf{q}_{2} .
\end{gathered}
$$

Using the cyclic property of the scalar triple product we can write

$$
\left[\mathbf{q}_{1} \times \mathbf{n}+\mathbf{f}_{0}^{T} \times \mathbf{q}_{2}\right] \cdot\left[\nabla G_{\delta} \times \mathbf{n}\right]=\mathbf{n} \times\left[\mathbf{q}_{1} \times \mathbf{n}+\mathbf{f}_{0}^{T} \times \mathbf{q}_{2}\right] \cdot \nabla G_{\delta} .
$$

Since both $\mathbf{f}_{0}^{T}$ and $\mathbf{q}_{2}$ are tangential vectors, $\mathbf{f}_{0}^{T} \times \mathbf{q}_{2}$ will contribute zero. Thus, we get

$$
\mathbf{n} \times \mathbf{q}_{1} \times \mathbf{n}=\mathbf{q}_{1}-\left(\mathbf{q}_{1} \cdot \mathbf{n}\right) \mathbf{n}=\mathbf{q}_{1}^{T},
$$

the tangential component of $\mathbf{q}_{1}$. The original integrand (without $\tau d \alpha$ ) becomes

$$
\begin{aligned}
& {\left[\left(\mathbf{f}^{T}(\alpha)-\mathbf{f}_{0}^{T}\right) \times \mathbf{n}(\alpha)+\mathbf{f}_{0}^{T} \times\left(\mathbf{n}(\alpha)-\mathbf{n}_{0}\right)\right] \cdot\left[\nabla G_{\delta} \times \mathbf{n}(\alpha)\right]} \\
& \approx \mathbf{q}_{1}^{T} \cdot \nabla G_{\delta}=\sum_{r=1}^{2}\left[\left(\frac{\partial \mathbf{f}^{T}}{\partial \alpha_{r}} \cdot \mathbf{T}_{1}^{*}\right) \mathbf{T}_{1}+\left(\frac{\partial \mathbf{f}^{T}}{\partial \alpha_{r}} \cdot \mathbf{T}_{2}^{*}\right) \mathbf{T}_{2}\right] \alpha_{r} \cdot \nabla G_{\delta} .
\end{aligned}
$$

Since $\mathbf{q}_{1}^{T}$ is tangential, we can use

$$
\nabla G_{\delta}=\sum_{j=1}^{2} \frac{\partial G_{\delta}}{\partial \alpha_{j}} \mathbf{T}_{j}^{*}+\text { normal part, }
$$

to write

$$
\left[\left(\mathbf{f}^{T}(\alpha)-\mathbf{f}_{0}^{T}\right) \times \mathbf{n}(\alpha)+\mathbf{f}_{0}^{T} \times\left(\mathbf{n}(\alpha)-\mathbf{n}_{0}\right)\right] \cdot\left[\nabla G_{\delta} \times \mathbf{n}(\alpha)\right] \approx \sum_{j, r}\left(\frac{\partial \mathbf{f}^{T}}{\partial \alpha_{r}} \cdot \mathbf{T}_{j}^{*}\right) \alpha_{r} \frac{\partial G_{\delta}}{\partial \alpha_{j}} .
$$

Further, suppose $\mathbf{y}=b \mathbf{n}_{0}$, for $\alpha$ near $0, \tau(\alpha) \approx \tau(0)=\tau$, and we replace $\mathbf{x}(\alpha)$ with its projection $J \alpha=\mathbf{T}_{1} \alpha_{1}+\mathbf{T}_{2} \alpha_{2}$ in the tangent plane, where $J=\partial \mathbf{x} / \partial \alpha(0)$. Then, as the first approximation to (5.1) we let

$$
\begin{aligned}
I^{0} & =\int_{\partial \Omega} \sum_{j, r=1}^{2}\left(\frac{\partial \mathbf{f}^{T}}{\partial \alpha_{r}} \cdot \mathbf{T}_{j}^{*}\right) \frac{\partial}{\partial \alpha_{j}} G_{\delta}\left(J \alpha-b \mathbf{n}_{0}\right) \alpha_{r} \tau d \alpha \\
& =\sum_{j, r=1}^{2}\left(\frac{\partial \mathbf{f}^{T}}{\partial \alpha_{r}} \cdot \mathbf{T}_{j}^{*}\right) \iint_{\mathbb{R}^{2}} \frac{\partial}{\partial \alpha_{j}} G_{\delta}\left(J \alpha-b \mathbf{n}_{0}\right) \alpha_{r} \zeta(\alpha) \tau d \alpha,
\end{aligned}
$$


where $\zeta(\alpha)$ is a cut-off function with $\zeta=1$ near $\alpha=0$, and $\partial \mathbf{f}^{T} / \partial \alpha_{r}$ and $\mathbf{T}_{j}^{*}$ are replaced by their values at $\alpha=0$.

Proposition 5.1. Let $S^{0}$ be the sum corresponding to (5.3) with $\alpha=j h-v h$. Then

$$
S^{0}-I^{0}=h \sum_{j, r=1}^{2} c_{j, r}\left(\frac{\partial \mathbf{f}^{T}}{\partial \alpha_{r}} \cdot \mathbf{T}_{j}^{*}\right)+O\left(h^{p}\right)
$$

for large $p$, where $c_{j, r}$ are given by (3.19).

Proof. First, define

$$
K_{\delta}^{(j)}(\alpha, b)=\frac{\partial}{\partial \alpha_{j}} G_{\delta}\left(J \alpha-b \mathbf{n}_{0}\right) .
$$

To find $c_{j, r}$, we need to compute the Fourier transform of

$$
K_{\delta}^{(j, r)}(\alpha, b)=K_{\delta}^{(j)}(\alpha, b) \alpha_{r}
$$

in $\alpha$ alone. The 3D Fourier transform of $G_{\rho}(\mathbf{x})=-\operatorname{erf}(|\mathbf{x}| / \rho) / 4 \pi|\mathbf{x}|$ is

$$
\hat{G}_{\rho}(\mathbf{k})=-(2 \pi)^{-3 / 2}|\mathbf{k}|^{-2} e^{-\rho^{2}|\mathbf{k}|^{2} / 4}, \quad \mathbf{k}=\left(k_{1}, k_{2}, k_{3}\right) \in \mathbb{R}^{3} .
$$

Since $G_{\rho}\left(J \alpha-b \mathbf{n}_{0}\right)$ is a radial function, it can be written as a composition $G_{\rho}\left(J \alpha-b \mathbf{n}_{0}\right)=$ $\left(G_{\rho} \circ M\right)(\alpha, b)$, where $M(\alpha, b)=(B \alpha, b), B=\left(J^{*} J\right)^{1 / 2},|B \alpha|^{2}=J^{*} J \alpha \cdot \alpha=\sum_{i j} g_{i j} \alpha_{i} \alpha_{j}$ (see [3]). The 3D transform of $G_{\rho} \circ M$ is then computed as

$$
\left(G_{\rho} \circ M\right)^{r}(\mathbf{k})=|\operatorname{det} M|^{-1} \hat{G}_{\rho}\left(\left(M^{*}\right)^{-1} \mathbf{k}\right)=\tau^{-1} \hat{G}_{\rho}\left(B^{-1}\left(k_{1}, k_{2}\right), k_{3}\right)=\tau^{-1} \hat{G}_{\rho}\left(l, k_{3}\right),
$$

where $l^{2}=\sum_{i, j=1}^{2} g^{i j} k_{i} k_{j}$. Therefore,

$$
\hat{K}_{\rho}^{(j)}(\mathbf{k})=\left(\frac{\partial}{\partial \alpha_{j}} G_{\rho} \circ M\right)^{\gamma}(\mathbf{k})=i k_{j} \tau^{-1} \hat{G}_{\rho}\left(l, k_{3}\right), \quad j=1,2,
$$

and the transform of $K_{\rho}^{(j)}(\alpha, b)$ in $\alpha$ alone is

$$
\begin{aligned}
K_{\rho}^{(j)}(\cdot, b)^{r}\left(k_{1}, k_{2}\right) & =(2 \pi)^{-1 / 2} \int_{-\infty}^{\infty} \hat{K}_{\rho}^{(j)}\left(k_{1}, k_{2}, k_{3}\right) e^{i k_{3} b} d k_{3} \\
& =-(2 \pi)^{-2} i k_{j} \tau^{-1} \int_{-\infty}^{\infty} \frac{1}{l^{2}+k_{3}^{2}} e^{-\rho^{2}\left(l^{2}+k_{3}^{2}\right) / 4} e^{i k_{3} b} d k_{3} \\
& =-(2 \pi)^{-2} i k_{j} \tau^{-1} e^{-\rho^{2} l^{2} / 4} \int_{-\infty}^{\infty} \frac{e^{-\rho^{2} k_{3}^{2} / 4}}{l^{2}+k_{3}^{2}} e^{i k_{3} b} d k_{3} \\
& =-(8 \pi \tau)^{-1} \frac{i k_{j}}{l}\left[e^{l b} \operatorname{erfc}(b / \rho+l \rho / 2)+e^{-l b} \operatorname{erfc}(-b / \rho+l \rho / 2)\right] \\
& =-\frac{i k_{j}}{8 \pi \tau l} E\left(\frac{b}{\rho}, \frac{l \rho}{2}\right),
\end{aligned}
$$


where $E$ was defined in (3.15). This is similar to (3.15) in [3]. Since $K_{\rho}^{(j, r)}=K_{\rho}^{(j)} \alpha_{r}$, we get an extra derivative in the Fourier transform,

$$
\begin{aligned}
\hat{K}_{\rho}^{(j, r)}\left(k_{1}, k_{2} ; b\right) & =i \frac{\partial}{\partial k_{r}} \hat{K}_{\rho}^{(j)}=\frac{1}{8 \pi \tau} \frac{\partial}{\partial k_{r}}\left[\frac{k_{j}}{l} E(b / \rho, l \rho / 2)\right] \\
& =\frac{1}{8 \pi \tau}\left[\frac{\partial}{\partial k_{r}}\left(\frac{k_{j}}{l}\right) E(b / \rho, l \rho / 2)+\frac{k_{j}}{l} \sum_{s=1}^{2} g^{r s} \frac{k_{s}}{l}\left(\frac{\partial}{\partial l} E(b / \rho, l \rho / 2)\right)\right] \\
& =\frac{1}{8 \pi \tau}\left[\frac{\delta_{j r}}{l} E(b / \rho, l \rho / 2)-\frac{k_{j}}{l^{2}}\left(\sum_{s=1}^{2} g^{r s} \frac{k_{s}}{l}\right) E(b / \rho, l \rho / 2)\right. \\
& +\frac{k_{j}}{l}\left(\sum_{s=1}^{2} g^{r s} \frac{k_{s}}{l}\right) b E_{-}(b / \rho, l \rho / 2)-\frac{k_{j}}{l}\left(\sum_{s=1}^{2} g^{r s} \frac{k_{s}}{l}\right) \frac{2 \rho}{\sqrt{\pi}} e^{-b^{2} / \rho^{2}} e^{\left.-l^{2} \rho^{2} / 4\right],}
\end{aligned}
$$

with $E_{-}$defined as in (3.20). Now, applying Lemma 3.1 from [3] to (5.3), we have the discretization error $S^{0}-I^{0}=\epsilon h+O\left(h^{p}\right)$, where

$$
\epsilon=2 \pi \tau \sum_{j, r=1}^{2}\left(\frac{\partial \mathbf{f}^{T}}{\partial \alpha_{r}} \cdot \mathbf{T}_{j}^{*}\right) \sum_{n \neq 0} e^{-2 \pi i n v} \hat{K}_{\rho}^{(j, r)}(2 \pi n, b / h) .
$$

(That the remainder is high order is evident from the proof of Lemma 3.1 in [2].) Letting $\lambda=b / \delta$ and $\|n\|=\sqrt{g^{i j} n_{i} n_{j}}$ in (5.7), this becomes

$$
\begin{aligned}
\epsilon & =\frac{1}{4} \sum_{j, r=1}^{2}\left(\frac{\partial \mathbf{f}^{T}}{\partial \alpha_{r}} \cdot \mathbf{T}_{j}^{*}\right) \sum_{n \neq 0} e^{-2 \pi i n v}\left[\frac{\delta_{j r}}{2 \pi\|n\|} E(\lambda, \pi \rho\|n\|)-\frac{n_{j}}{2 \pi\|n\|^{2}}\left(\sum_{s=1}^{2} g^{r s} \frac{n_{s}}{\|n\|}\right) E(\lambda, \pi \rho\|n\|)\right. \\
& \left.+\frac{n_{j}}{\|n\|}\left(\sum_{s=1}^{2} g^{r s} \frac{n_{s}}{\|n\|}\right) \lambda \rho E_{-}(\lambda, \pi \rho\|n\|)-\frac{n_{j}}{\|n\|}\left(\sum_{s=1}^{2} g^{r s} \frac{n_{s}}{\|n\|}\right) \frac{2 \rho}{\sqrt{\pi}} e^{-\lambda^{2}} e^{-\pi^{2}\|n\|^{2} \rho^{2}}\right] .
\end{aligned}
$$

Combining the terms with $\pm n$, we get $\epsilon=\sum_{j, r=1}^{2} c_{j, r} F_{r}^{j}$, where $c_{j, r}$ are given by (3.19). Therefore, the correction for $\sigma$ th patch is (3.18).

It remains to establish the estimate for $\epsilon_{2}$ in Theorem 3.1. Since we have just found the quadrature error in the simplified integral $I^{0}$ of (5.3), $\epsilon_{2}$ amounts to the quadrature error in the remainder $I-I^{0}$, with $I$ as in (5.1). It can be estimated by applying Lemma 3.1 of [3] to the quadrature of the leading contributions to $I-I^{0}$; see page 611 of [3].

\section{Numerical examples}

Our first example will demonstrate the corrections for the tangential integral. We compute $v^{\tau}(\mathbf{y})$ from (3.6) along the sphere with unit radius with $\mathbf{f}=(3 / 2,0,0)$, evaluated at the point $\mathbf{y}=(1,0,0)$. The exact value of the integral is 1 . Analogous to [3], we represent 
the surface $\partial \Omega$ of the sphere $x_{1}^{2}+x_{2}^{2}+x_{3}^{2}=1$ using two stereographic projections on the equatorial plane by rays through the south $(0,0,-1)$ and the north $(0,0,1)$ poles. The first gives a coordinate system, $X^{1}: \mathbb{R}^{2} \rightarrow U^{1}=\partial \Omega-\{(0,0,-1)\}$ :

$$
x_{1}=\frac{2 \alpha_{1}}{1+|\alpha|^{2}}, \quad x_{2}=\frac{2 \alpha_{2}}{1+|\alpha|^{2}}, \quad x_{3}=\frac{1-|\alpha|^{2}}{1+|\alpha|^{2}}
$$

while the other gives a system $X^{2}: \mathbb{R}^{2} \rightarrow U^{2}=\partial \Omega-\{(0,0,1)\}$ as in (6.1) but with $x_{3} \rightarrow-x_{3}$. We consider discs of radius 1.25 in the plane, so that the two systems overlap, and define the partition of unity as in [3], by setting first $\phi^{\sigma}\left(X^{\sigma}(\alpha)\right)=\exp \left(-1.25^{2} /\left(1.25^{2}-|\alpha|^{2}\right)\right)$ for $|\alpha| \leq 1.25$ and $\phi^{\sigma}=0$ otherwise, so that $\phi^{\sigma}$ is smooth and has the support $\left\{X^{\sigma}(\alpha):|\alpha| \leq 1.25\right\}$. Then the partition of unity $\left\{\psi^{1}, \psi^{2}\right\}$ is defined by $\psi^{\sigma}(x)=\phi^{\sigma}(x) /\left(\phi^{1}(x)+\phi^{2}(x)\right)$.

The integral is first approximated by the sum $S^{\tau}$ in (3.10), then the corrected solution is obtained by adding the correction for regularization $\mathcal{T}_{1}$, defined in (3.16), and for discretization $\mathcal{T}_{2}^{\sigma}$, defined in (3.18). The geometric quantities and surface derivatives were computed analytically. We choose a grid size $h$ in the coordinate systems and let $\delta=\rho h$. Figures 1 and 2 show the error, computed as $\left|v^{\tau}(\mathbf{y})-S^{\tau}\right|$, labeled 'not corrected', and $\left|v^{\tau}(\mathbf{y})-\left(S^{\tau}+\mathcal{T}_{1}+\sum_{\sigma} \mathcal{T}_{2}^{\sigma}\right)\right|$, labeled 'corrected'. In Figure 1, we set $h=1 / 32$ and vary $\delta / h=0.01, \ldots, 10$ to see the effect of the two sources of error, regularization and discretization. For larger values of $\delta / h$ (typically, 1 and higher), the regularization error is dominant. The discretization error is small, and the terms in the infinite sum (3.19) in $\mathcal{T}_{2}$ decay very fast. When $\delta / h$ is small, the discretization error dominates, and many terms in the sum (3.19) are needed to reduce it. Figure 2 shows the convergence rates of the solutions for varying discretization size $h$. Regularization was set to $\delta=0.01 h$ (left graph) and $\delta=h$ (right graph). Both show the errors are first order without corrections, and corrections improve the accuracy to third order. To compute the sum (3.19), $n_{\max }=200$ terms were taken when $\delta / h=0.01$, and only $n_{\max }=2$ terms were used when $\delta / h=1$. It is therefore more desirable to choose values of $\delta / h$ at 1 or higher in practice.

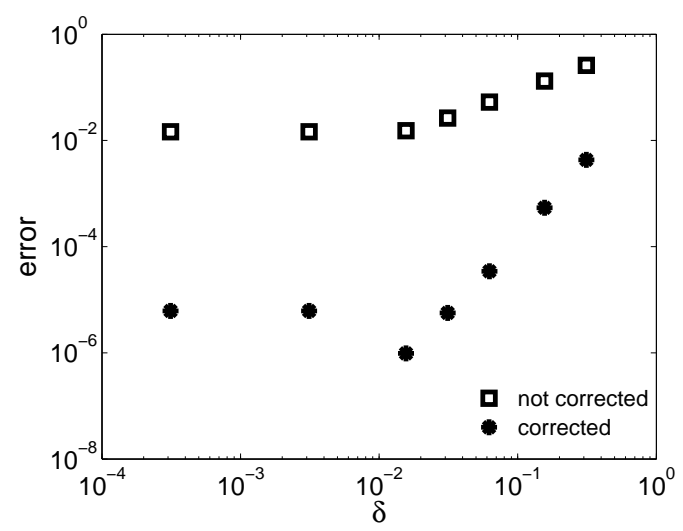

Figure 1: Tangential integral. Error at $(1,0,0)$ for $\delta=\rho h$ with $h=1 / 32$. 

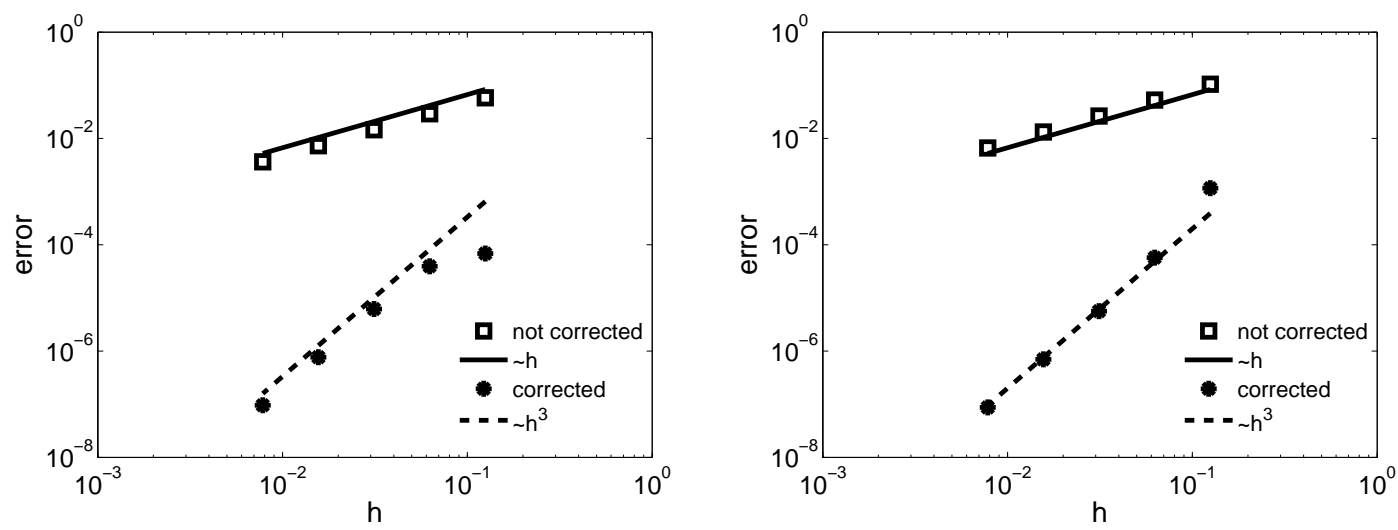

Figure 2: Tangential integral. Error at $(1,0,0)$ with $\delta=0.01 h$ and $\delta=h$.

For our second example, we compute the well-known Stokes flow around a solid sphere of unit radius translating with velocity $\mathbf{U}=(U, 0,0)$, where we set $U=1$. We compute the pressure and velocity defined in (2.4), where the hydrodynamic traction on the sphere is $-\mathbf{f}=-3 \mathbf{U} / 2$. The integrals are first approximated by sums in (3.9) and (3.10). To obtain the corrected solution, we add corrections as in (3.21). For a chosen grid size $h$, the regularization is set to $\delta=2 h$. To compute the nearly singular integrals, we cover $\mathbb{R}^{3}$ with a three-dimensional mesh of size $h$, and select points $\mathbf{y}$ outside the sphere that are within distance $h$ from the surface. As a comparison, we also compute the flow using the regularized Stokeslets [7],

$$
\begin{aligned}
& \mathbf{u}^{R S}(\mathbf{y})=\sum_{j, \sigma}\left[\frac{\mathbf{f}\left(\mathbf{x}_{j}^{\sigma}\right)}{8 \pi} \frac{r_{j \sigma}^{2}+2 \delta^{2}}{\left(r_{j \sigma}^{2}+\delta^{2}\right)^{3 / 2}}+\frac{1}{8 \pi}\left[\mathbf{f}\left(\begin{array}{l}
\sigma \\
j
\end{array}\right) \cdot\left(\mathbf{y}-\mathbf{x}_{j}^{\sigma}\right)\right] \frac{\mathbf{y}-\mathbf{x}_{j}^{\sigma}}{\left(r_{j \sigma}^{2}+\delta^{2}\right)^{3 / 2}}\right] \psi_{j}^{\sigma} A_{j}^{\sigma} h^{2}, \\
& p^{R S}(\mathbf{y})=\sum_{j, \sigma} \frac{\mathbf{f}\left(\mathbf{x}_{j}^{\sigma}\right) \cdot\left(\mathbf{y}-\mathbf{x}_{j}^{\sigma}\right)}{\left(r_{j \sigma}^{2}+\delta^{2}\right)^{3 / 2}} \psi_{j}^{\sigma} A_{j}^{\sigma} h^{2},
\end{aligned}
$$

where $r_{j \sigma}=\left|\mathbf{y}-\mathbf{x}_{j}^{\sigma}\right|$. The error in each approximation is defined as

$$
\begin{aligned}
& \operatorname{error}(\mathrm{u})=\max _{\mathbf{y}}|| \mathbf{u}_{\text {computed }}(\mathbf{y})-\mathbf{u}_{\text {exact }}(\mathbf{y}) \|_{2}, \\
& \operatorname{error}(\mathrm{p})=\max _{\mathbf{y}}\left|p_{\text {computed }}(\mathbf{y})-p_{\text {exact }}(\mathbf{y})\right|,
\end{aligned}
$$

where the exact velocity and pressure fields outside the sphere are

$$
\begin{aligned}
u_{i}(\mathbf{y}) & =\frac{1}{4 r}\left(3+\frac{1}{r^{2}}\right) U_{i}+\frac{3}{4 r}\left(1-\frac{1}{r^{2}}\right) \frac{y_{i} y_{j}}{r^{2}} U_{j}, \\
p(\mathbf{y}) & =p_{0}+\frac{3}{2 r} \frac{\mathbf{U} \cdot \mathbf{y}}{r^{2}},
\end{aligned}
$$

with $p_{0}$ an arbitrary constant and $r$ the distance from the point $\mathbf{y}$ to the center of the sphere. The errors, shown in Figure 3, are only first order without corrections, and display the combined order $O\left(h^{3}\right)$ in the corrected case. 

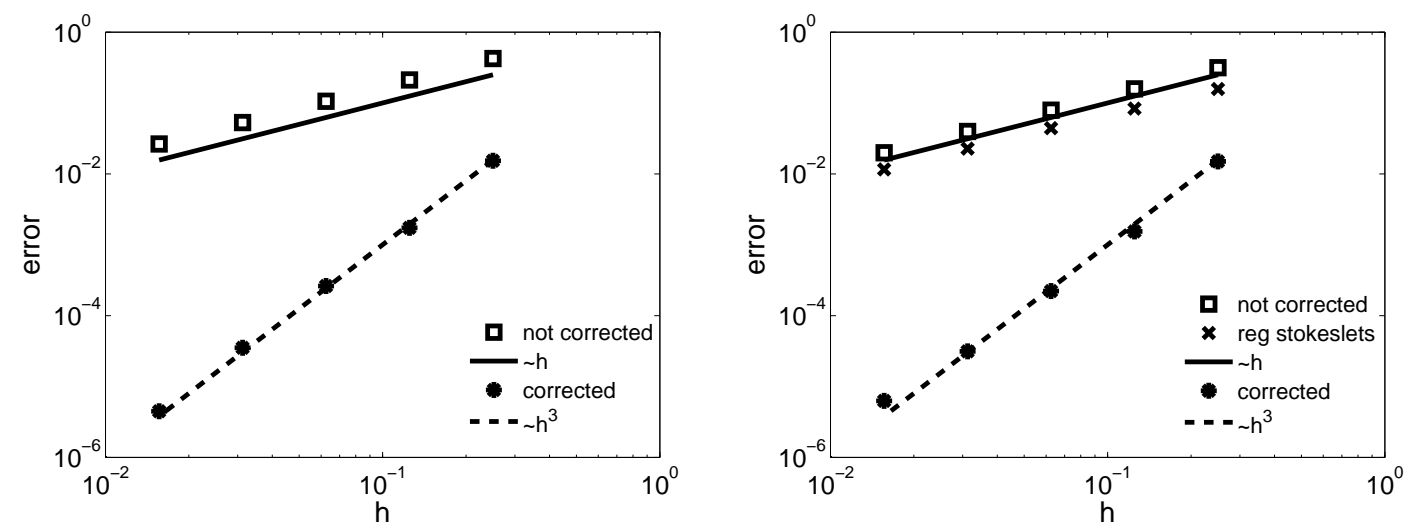

Figure 3: Stokes flow around sphere. Error at nearby points: (a) pressure, (b) velocity.

For our third example, we consider the ellipsoid $x_{1}^{2}+x_{2}^{2}+x_{3}^{2} / 2=1$. We use coordinate systems as in (6.1), with $x_{3}$ multiplied by $\sqrt{2}$. To compute the surface derivatives, we use a cubic polynomial interpolation in $\alpha$, although quadratic interpolation seems to suffice as well. To estimate the order of accuracy at a nearby point $\mathbf{y}=(1+h / 2,0,0)$, we define the error as

$$
\begin{aligned}
& \operatorname{error}(\mathbf{u})=|| \mathbf{u}_{h}(\mathbf{y})-\mathbf{u}_{h / 2}(\mathbf{y}) \|_{2} \sim O\left(h^{q}\right), \\
& \operatorname{error}(\mathrm{p})=\left|p_{h}(\mathbf{y})-p_{h / 2}(\mathbf{y})\right| \sim O\left(h^{q}\right),
\end{aligned}
$$

where $q$ is expected to be 1 without corrections and 3 with corrections. The results with $\delta=2 h$ are shown in Figure 4 and confirm the expected rates.
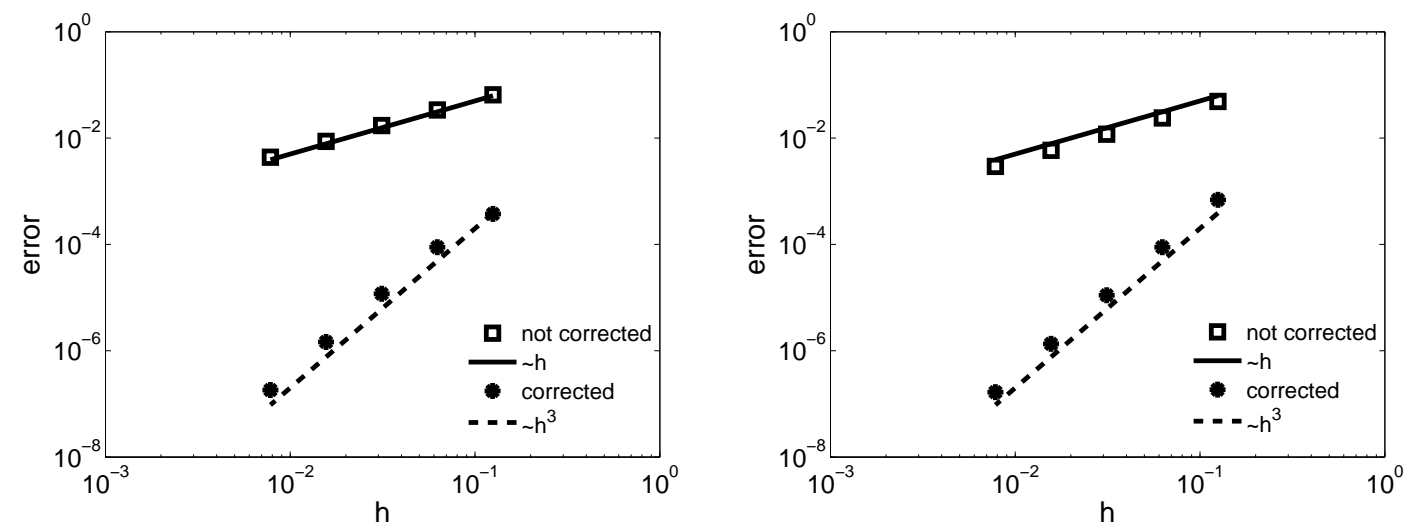

Figure 4: Stokes flow around ellipse. Error at $(1+\mathrm{h} / 2,0,0)$ : (a) pressure, (b) velocity. 


\section{Acknowledgements}

Research of the second author was supported in part by the National Science Foundation under Grant DMS-0806482.

\section{References}

[1] R. Aris, Vectors, Tensors, and the Basic Equations of Fluid Mechanics, Dover, New York, 1962.

[2] J. T. Beale and M.-C. Lai, A method for computing nearly singular integrals, SIAM J. Numer. Anal., 38 (2001), 1902-1925.

[3] J. T. Beale, A grid-based boundary integral method for elliptic problems in three dimensions, SIAM J. Numer. Anal., 42 (2004), 599-620.

[4] O. P. Bruno and L. A. Kunyansky, A fast, high-order algorithm for the solution of surface scattering problems: basic implementation, tests, and applications, J. Comput. Phys., 169 (2001), 80-110.

[5] D. Colton and R. Kress, Integral Equation Methods in Scattering Theory, Wiley, New York, 1983.

[6] R. Cortez, The method of regularized Stokeslets, SIAM J. Sci. Comput., 23(4) (2001), 12041225.

[7] R. Cortez, L. Fauci and A. Medovikov, The method of regularized Stokeslets in three dimensions: Analysis, validation, and application to helical swimming, Phys. Fluids, 17 (2005), $1-14$.

[8] H. Flores, E. Lobaton, S. Méndez-Diez, S. Tlupova, and R. Cortez, A study of bacterial flagellar bundling, Bull. Math. Biol., 67 (2005), 137-168.

[9] L. Greengard, M. C. Kropinski, and A. Mayo, Integral equation methods for Stokes flow and isotropic elasticity in the plane, J. Comp. Phys., 125 (1996), 403-414.

[10] J. Helsing and R. Ojala, On the evaluation of layer potentials close to their sources, J. Comput. Phys., 227 (2008), 2899-2921.

[11] G. C. Hsiao and W. L. Wendland, Boundary Integral Equations, Springer-Verlag, Berlin, 2008.

[12] P. R. Johnston, Application of sigmoidal transformations to weakly singular and nearsingular boundary element integrals, Int. J. Numer. Meth. Eng., 45(10) (1999), 1333-1348.

[13] R. Kress, Linear Integral Equations, Springer-Verlag, New York, 2nd edition, 1999.

[14] J. Lighthill, Helical distributions of Stokeslets, J. Eng. Math., 30 (1996), 35-78.

[15] M. J. Nicholas, A higher order numerical method for 3-d doubly periodic electromagnetic scattering problems, Commun. Math. Sci., 6(3) (2008), 669-694.

[16] C. Pozrikidis, Boundary Integral and Singularity Methods for Linearized Viscous Flow, Cambridge University Press, Cambridge, 1992.

[17] C. Pozrikidis, Introduction to Theoretical and Computational Fluid Dynamics, Oxford University Press, Oxford, 1997.

[18] C. Pozrikidis, Interfacial dynamics for Stokes flow, J. Comput. Phys., 169 (2001), 250-301.

[19] A. Sierou and J. F. Brady, Accelerated Stokesian Dynamics simulations, J. Fluid Mech., 448 (2001), 115-146.

[20] S. Tlupova and R. Cortez, Boundary integral solutions of coupled Stokes and Darcy flows, J. Comput. Phys., 228(1) (2009), 158-179. 
[21] S. K. Veerapaneni, A. Rahimian, G. Biros, and D. Zorin, A fast algorithm for simulating vesicle flows in three dimensions, J. Comput. Phys., 230(14) (2011), 5610-5634.

[22] L. Ying, G. Biros, and D. Zorin, A high-order 3D boundary integral equation solver for elliptic PDEs in smooth domains, J. Comput. Phys., 219 (2006), 247-275.

[23] W. Ying and J. T. Beale, A fast accurate boundary integral method for potentials on closely packed cells, submitted to Commun. Comput. Phys., 2012.

[24] A. Z. Zinchenko, M. A. Rother, and R. H. Davis, Cusping, capture, and breakup of interacting drops by a curvatureless boundary-integral algorithm, J. Fluid Mech., 391 (1999), 249292. 